\title{
NATF (Native and Tissue-Specific Fluorescence): A Strategy for Bright, Tissue-Specific GFP Labeling of Native Proteins in Caenorhabditis elegans
}

\author{
Siwei He, ${ }^{*+}$ Andrea Cuentas-Condori, ${ }^{\dagger}$ and David M. Miller, III*,t,1 \\ *Program in Neuroscience, Vanderbilt University, Nashville, Tennessee and 'Department of Cell and Developmental Biology, \\ Vanderbilt University School of Medicine, Nashville, Tennessee 37240 \\ ORCID IDs: 0000-0002-4847-0031 (A.C.-C.); 0000-0001-9048-873X (D.M.M.)
}

\begin{abstract}
GFP labeling by genome editing can reveal the authentic location of a native protein, but is frequently hampered by weak GFP signals and broad expression across a range of tissues that may obscure cell-specific localization. To overcome these problems, we engineered a Native And Tissue-specific Fluorescence (NATF) strategy that combines genome editing and split-GFP to yield bright, cellspecific protein labeling. We use clustered regularly interspaced short palindromic repeats CRISPR/Cas 9 to insert a tandem array of seven copies of the GFP11 $\beta$-strand $\left(g f p 11_{x 7}\right.$ ) at the genomic locus of each target protein. The resultant $g f p 11_{x 7}$ knock-in strain is then crossed with separate reporter lines that express the complementing split-GFP fragment (gfp1-10) in specific cell types, thus affording tissue-specific labeling of the target protein at its native level. We show that NATF reveals the otherwise undetectable intracellular location of the immunoglobulin protein OIG-1 and demarcates the receptor auxiliary protein LEV-10 at cell-specific synaptic domains in the Caenorhabditis elegans nervous system.
\end{abstract}

KEYWORDS C. elegans; cell specificity; genome editing; GFP; NATF; native expression; protein localization

$R^{2}$ ELIABLE localization of a given protein can provide useful clues to its mechanism of action. One way to achieve this goal is to label the protein of interest with tags, such as fluorescent proteins (e.g., GFP) (Chalfie 2009; Remington 2011) or small peptides (e.g., FLAG or HA) (Terpe 2003). Because tagged proteins are typically expressed with heterologous promoters or from multicopy transgenic arrays, this approach can result in misleading signals due to overexpression (Praitis et al. 2001). This problem can be obviated by using clustered regularly interspaced short palindromic repeats (CRISPR)/Cas9 for single-copy labeling of the native protein (Hsu et al. 2014; Dickinson et al. 2015), but this genome-editing strategy suffers from two additional limitations. First, the endogenous expression level of a target protein may be too low for detection. Second, the protein of

Copyright (C) 2019 by the Genetics Society of America doi: https://doi.org/10.1534/genetics.119.302063

Manuscript received February 27, 2019; accepted for publication March 28, 2019; published Early Online April 5, 2019.

Available freely online through the author-supported open access option

Supplemental material available at https://doi.org/10.25386/genetics.7898075.

${ }^{1}$ Corresponding author: Department of Cell and Developmental Biology, Vanderbilt

University Medical Center, Vanderbilt University School of Medicine, 3120 MRBIII,

Nashville, TN 37232-8240. E-mail: david.miller@vanderbilt.edu interest may be expressed in several tissues, thus preventing a clear delineation of cell-specific localization. Previous efforts have focused on solving each of these problems separately. For example, strategies to enhance detection include the use of brighter fluorescent proteins (El Mouridi et al. 2017; Hostettler et al. 2017) and the development of the SunTag label, which uses a scaffold-like structure to recruit multiple copies of GFP (Tanenbaum et al. 2014). The "FLP-on" strategy uses cell-specific FLP drivers to activate GFP expression from CRISPR/Cas9-engineered FRT sites but may not yield a visible signal for low-expressing genes (Schwartz and Jorgensen 2016). Here, we describe an experimental approach, NATF (Native And Tissue-specific Fluorescence or "Native"), that exploits a combinatorial strategy to achieve both bright and cellspecific labeling of the protein of interest.

Our approach relies on the finding that the barrel-like GFP structure can be reconstituted by the spontaneous interaction of two separate GFP peptides derived from the highly stable GFP variant, superfolder GFP. The larger of these fragments is comprised of the first $10 \beta$-strands (GFP1-10). Its smaller complement, a short, 16 amino acid sequence, contains the 11th $\beta$-strand (GFP11) (Cabantous et al. 2005). The reconstituted 
split-GFP hybrid produces a fluorescent signal that is substantially brighter than weak background fluorescence arising from overexpression of the GFP1-10 fragment (Feng et al. 2017). Thus, to enhance the GFP signal, a target protein can be tagged with multiple copies of the short GFP11 peptide and then coexpressed with excess GFP1-10 (Kamiyama et al. 2016; Feng et al. 2017). In addition to labeling the native protein with smaller covalent tags, this combinatorial approach offers the further benefit of limiting the GFP signal to the specific cell type in which GFP1-10 is expressed (Figure 1A).

In this report, we describe a NATF toolbox that combines split-GFP and CRISPR technology for live-cell imaging of labeled Caenorhabditis elegans proteins expressed at native levels. With this approach, a GFP11 multicopy DNA array (GFP11 ${ }_{\mathrm{X} 7}$ ) is inserted into the target gene. The resultant knock-in strain can then be crossed with separate reporter lines in which GFP1-10 is expressed in different cell types for tissue-specific visualization of the reconstituted NATF GFP (Figure 1A). We utilized this strategy for effective enhancement of an otherwise weak signal from single-copy labeling of a key protein (OIG-1) as well as the cell-specific resolution of a receptor accessory protein (LEV-10) at closely spaced but functionally distinct synapses in the $C$. elegans nervous system.

\section{Materials and Methods}

\section{C. elegans strains}

C. elegans strains were maintained at room temperature on NGM plates seeded with OP50 (Brenner 1974). Some strains were obtained from the Caenorhabditis Genetics Center (CGC). The N2 Bristol strain was used as the wild-type reference. Transgenic animals were generated using standard microinjection techniques (Evans 2006). Strains used in this study are described in Supplemental Material, Table S1.

\section{Molecular biology}

single-guide RNA/Cas9 plasmid design: A 200 bp DNA sequence that contained the desired cut site was submitted to the optimized CRISPR Design online tool (http://crispr.mit. edu/) to predict single-guide RNA (sgRNA) sequences. To enhance gene-editing efficiency, we selected a 5' $\mathrm{N}_{18}$ GGNGG sequence (Farboud and Meyer 2015) as an sgRNA targeting site for both oig-1 and lev-10. For oig-1, 5'-GGAGAGAAAGAC GAAAATGG-3' was cloned into pDD162 (\#47549; Addgene), a plasmid that contains the sgRNA backbone and Cas9 expression system, using Q5 site-directed mutagenesis (New England Biolabs, Beverly, MA). Similarly, for lev-10, 5'-ACGAATCGA CTGGTGGCCGG-3' was used as the sgRNA target sequence, which is $\sim 80$ bp upstream of the lev-10 stop codon.

CRISPR repair template for oig-1 and lev-10: To create the Self-Excising drug selection Cassette (SEC) SEC repair template for oig-1 TagRFP (Tag red fluorescent protein) CRISPR knock-in, flanking 500-bp genomic DNA regions immediately upstream and downstream of the desired insertion site were amplified by PCR using the following primers (Primer 1 and Primer 2 for the upstream homology arm, and Primer 3 and Primer 4 for the downstream homology arm) with overlap regions to the target plasmid pDD284 (\#66825; Addgene):

OIG-1 Primer 1: 5'-GACGTTGTAAAACGACGGCCAGTCGA CCTAACCATTCCAAAAGAT-3'.

OIG-1 Primer 2: 5'-TGAGCTCCTCTCCCTTGGAGACCATCG CATTTATTCCAACTGATA-3'.

OIG-1 Primer 3: 5'-TTACAAGGATGACGATGACAAGAGAA AATCTTCGCATATAGAAGA-3'.

OIG-1 Primer 4: 5'-CAGGAAACAGCTATGACCATGTTATCC AAGTCGGAGTACTGTTCA-3'.

The amplified DNA fragments were cloned into plasmid pDD284 using Gibson cloning (New England Biolabs) to create the repair template. The corresponding protospacer adjacent motif (PAM) PAM sequence in the repair template plasmid was mutated from AGG to CCC using Q5 site-directed mutagenesis to produce the final plasmid, pSH30. Correct insertions and mutations were confirmed by sequencing.

To create the SEC repair template (pSH55) for the oig-1 GFP $11_{\mathrm{x} 7}$ CRISPR knock-in, the GFP1 $11_{\mathrm{x} 7}$ coding sequence was amplified from a previously published plasmid (\#70224; Addgene) and inserted into pSH30 to replace the TagRFP sequence by In-Fusion cloning (Takara) with the following primers.

Fragment.FOR 221 5'-GTTGGAATAAATGCGATGCGTGACC ACATGGTCCTT-3'.

Fragment.REV 222 5'-AAAGTACAGATTCTCGGTGATACCG GCAGCAT-3'.

Vector.FOR $223 \quad 5^{\prime}$-GAGAATCTGTACTTTCAATCCGGAAA GGTAAG-3'.

Vector.REV 224 5'-CGCATTTATTCCAACTGATAGAAAGCAT AAAAGTAGT-3'.

To create the GFP and GFP $11_{x 7}$ knock-in repair template for LEV-10, we used a two-step In-Fusion cloning method. Next, $\sim 500$ bp of DNA sequences upstream and downstream of the lev-10 stop codon were selected for flanking homology arms. DNA was amplified, and then sequentially cloned into pSH30 or pSH55 to replace the original oig- 1 homology arms. The resultant plasmids were then used as templates for site-direct mutagenesis to create the final repair template plasmid with sgRNA-binding sequences mutated, pSH84 (GFP knock-in), and pSH85 (GFP11 1 x knock-in). The primers for these cloning steps were designed with a strategy similar that used for oig-1 as described above. Primer sequences are available on request.

GFP1-10 reporter plasmids: The DNA sequence of GFP1-10 was amplified from pcDNA3.1-GFP1-10 (\#70219; Addgene) and cloned into pGH8 (Prab-3::mCherry) using In-Fusion cloning to create pSP1(Prab-3::gfp1-10). The Dorsal D (DD) and Ventral D (VD) DD and VD $\gamma$-aminobutyric acid (GABA)ergic neuron-specific promoter Pttr-39, the cholinergic-specific 


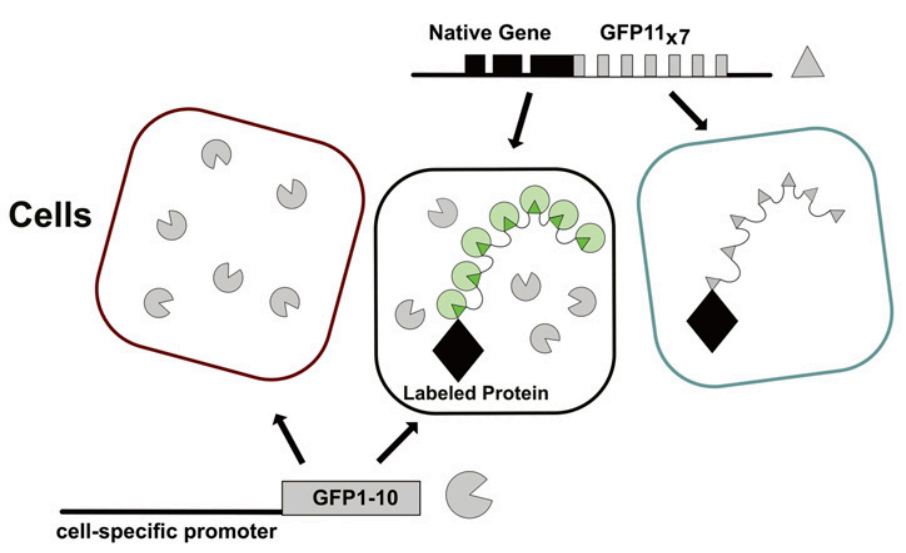

B

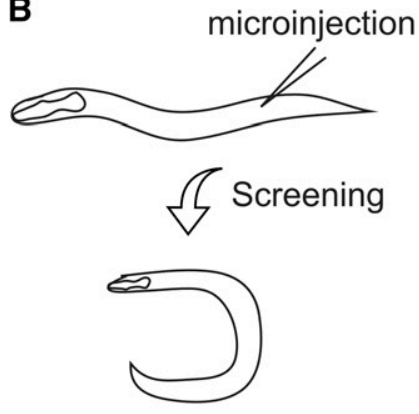

Hygromycin resistant + Roller

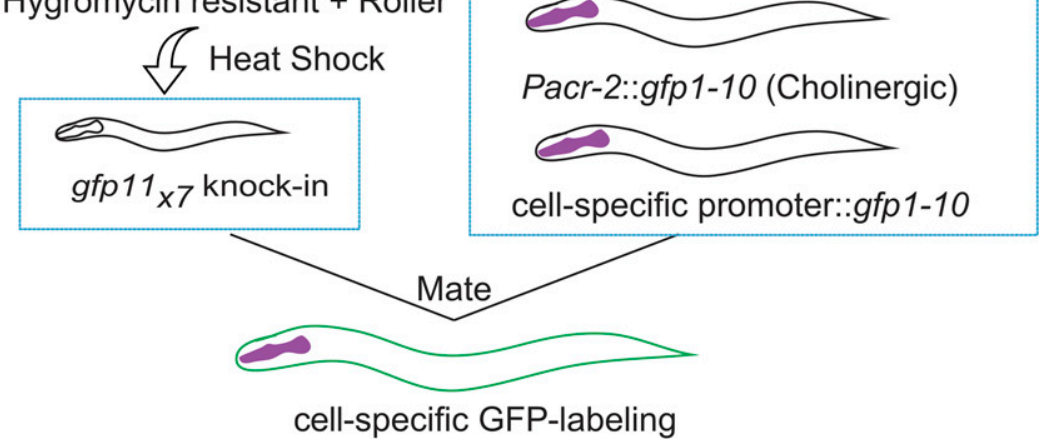

Figure 1 Robust, tissue-specific labeling of a target protein at its native expression level. (A) NATF. CRISPR/Cas9mediated gene editing is used to label a protein of interest with seven copies of GFP11 (GFP11 1 ). Transgenic expression of GFP1-10 with cell-specific promoters results in a bright, stable NATF fluorescent signal from multiple, reconstituted GFP molecules in specific tissues. (B) NATF workflow. Worms are injected with sgRNA, repair template, and co-injection markers. gfp $11_{x 7}$ knock-in worms are recovered after heat shock-induced excision of positive-selection genes. Crossing the gfp $11_{x 7}$ knock-in with gfp 1-10 reporter lines results in tissue-specific labeling of the target protein with a reconstituted NATF-GFP tag. CRISPR, clustered regularly interspaced short palindromic repeats; GABA, $\gamma$-aminobutyric acid; NATF, Native And Tissue-specific Fluorescence; sgRNA, single-guide RNA. promoter Pacr-2, and the muscle-specific Pmyo-3 promoter were amplified to replace the Prab-3 promoter in PSP1 to create pSH79 (Pttr-39::gfp1-10), pSH88 (Pacr-2::gfp1-10), pSH86 (Pmyo-3::gfp1-10), and pSH87 (Pflp-13::gfp1-10). To create a secreted GFP1-10 construct, In-Fusion cloning was used to add the first $114 \mathrm{bp}$ of the oig-1 sequence including the signal sequence (Philbrook et al. 2018) prior to the start codon of GFP1-10 in pSP1. The combined sequence was analyzed using the SignalP 4.1 Server to confirm that the predicted signal peptide was intact. The final plasmid, pSH69 (Prab-3::ssGFP1-10), was confirmed by sequencing.

\section{Confocal microscopy and image processing}

Fluorescent images were captured at room temperature using a Nikon (Garden City, NY) A1R confocal microscope. Nematodes were immobilized with $15 \mathrm{mM}$ levamisole/0.05\% tricaine on a $2 \%$ agarose pad in M9 buffer. All images for ACR-12::GFP fluorescence quantification were obtained with the same settings using a $40 \times / 1.4$ oil objective and Nyquist sampling. Constant laser power was used to compare the LEV-10::GFP fluorescence intensity to that of the NATF GPF signal produced by the combination of LEV-10::GFP11 $1_{x 7}$ with Pmyo-3::GFP1-10. Images in Figure 4 were three-dimensionally deconvolved with NIS-Elements with the automatic algorithm. For other images, ND2 files generated with NISElements were imported into Fiji for analysis. Maximum intensity projections were generated by selecting stacks that had both ventral and dorsal signals. Line scans of dorsal and ventral cords (Figure S2, B-D), and of the nerve ring (Figure 3C), were adjusted by subtraction of background fluorescence measured from an adjacent region for comparison of fluorescence intensities between samples. To compare the stability of GFP signals in lev-10::gfp vs. lev-10::gfp11 $x$; 
Pmyo-3::GFP1-10 strains, a region of interest (ROI) of the same size in each strain was bleached with a 405-nm laser for $15 \mathrm{sec}$ at $50 \%$ laser power. Images of the ROI were collected and compared, before and after photobleaching. OIG$1:: G F P 11_{x 7}$ and mCherry::OIG-1 strains were imaged using an A1R Nikon laser confocal to obtain intensity profiles (Figure $2 \mathrm{~A}$ and Figure $\mathrm{S} 3$ ). Fiji was used to draw $15-\mu \mathrm{m}$ long-line scans on the ventral cord and extract intensity profiles, which were exported to Excel. Fluorescent intensities were normalized to the maximum intensity value of each line scan. Intensities were plotted using Prism 6 software. Peaks exceeding a threshold of $75 \%$ of the normalized intensity for each sample were counted.

\section{AiryScan imaging}

Worms were mounted on $10 \%$ agarose pads and immobilized with $15 \mathrm{mM}$ levamisole/0.05\% tricaine dissolved in M9. A Zeiss ([Carl Zeiss], Thornwood, NY) LSM880 microscope equipped with an AiryScan detector and a $63 \times / 1.40$ PlanApochromat oil objective lens was used to acquire superresolution images of the DD neuron (Figure 4E). Images were acquired as a Z-stack $(0.19 \mu \mathrm{m} / \mathrm{step})$, spanning the total volume of the DD neuron and submitted for AiryScan image processing by ZEN software.

\section{Statistical analysis}

For all experiments, sample numbers were $n>10$. The Student's $t$-test was used for comparison between two groups. $P<0.05$ was considered significant. Prism 6 was used for statistical analysis.

\section{Immunoblotting}

OIG-1 CRISPR and transgenic overexpression strains were cultured on NGM plates seeded with OP50. Mixed-stage worms were collected into a $1.5-\mathrm{ml}$ tube by washing them off NGM plates with M9 buffer and pelleted by centrifugation. Next, $50 \mu \mathrm{l}$ of $2 \times$ SDS-PAGE protein sample buffer was added to a $50 \mu \mathrm{l}$ pellet of each genotype and heated to $90^{\circ}$ for $15 \mathrm{~min}$. Samples were centrifuged at $8000 \mathrm{rpm}$ for $5 \mathrm{~min}$ to remove debris (Miller et al. 1983). Then, $30 \mu \mathrm{l}$ of supernatant from each genotype was loaded on a $10 \%$ protein gel to run at $110 \mathrm{~V}$ for $60 \mathrm{~min}$, before being transferred to a PVDF membrane for immunoblot analysis (Duerr 2006). Immunoblots were treated with monoclonal anti-FLAG M2 antibody (1:500) (Sigma [Sigma Chemical], St. Louis, MO) to the 3XFLAG peptide followed by HRP-conjugated goat anti-mouse IgG antibody (1/2000), and soaked for 2 min in developing solution for chemiluminescent detection (Alegria-Schaffer et al. 2009).

\section{Data availability}

All reagents and C. elegans strains described in this work are available on request. Plasmids will be deposited at Addgene and $C$. elegans strains submitted to the CGC stock center. Supplemental figures have been uploaded at the Genetics Society of America Figshare portal. Supplemental material available at https://doi.org/10.25386/genetics.7898075.

\section{Results}

\section{Tool box and strategy for NATF GFP labeling}

We used a previously described CRISPR/Cas9 system for genome editing in C. elegans (Dickinson et al. 2015). In this approach, homology arms flank a self-excising cassette that carries positive selection markers (sqt-1) for the identification of transgenic worms ("rollers") and drug resistance (hygR) for the detection of CRISPR/Cas9-induced integrants. A brief heat-shock treatment induces excision of the marker cassette to restore wild-type movement ("nonroller") (Figure 1B). For split-GFP experiments, we replaced the fluorescent protein sequence in the original repair template plasmid with a gfp $11_{x 7}$ insert (Kamiyama et al. 2016). Homology arms of $\sim 500$ bp were used for the two genes targeted (oig-1 and lev-10) in this study (Figure S1A). We also constructed separate plasmids for expressing GFP1-10 in specific cell types including body muscles, all neurons, cholinergic neurons, and GABAergic neurons (Figure $1 \mathrm{~B}$ and Figure S1B). In these lines, the GFP1-10 transgenes are carried as extrachromosomal arrays that are maintained by selecting for a pharyngeal co-injection marker (Pmyo-2::mCherry) (Figure 1B). Cell-specific drivers are flanked with multiple cloning sites to facilitate the construction of plasmids for GFP1-10 expression in other tissues (Figure S1B). $g f p 11_{\mathrm{x} 7}$ knock-in strains can be confirmed within 2 weeks of the initial injection and then crossed with GFP1-10-expressing lines for characterization (Figure 1B).

\section{NATF GFP labeling reveals the intracellular localization of OIG-1 in GABAergic motor neurons}

oig-1 encodes a soluble protein with a single immunoglobulin domain (Figure 2B) that is temporally regulated in GABAergic motor neurons to antagonize a synaptic remodeling program; in oig-1 mutants, a postsynaptic acetylcholine receptor (AChR) containing the AChR subunit, ACR-12::GFP, is ectopically relocated from dorsal to ventral GABAergic neuron processes. OIG-1 is secreted when overexpressed from multicopy transgenic arrays to produce bright puncta adjacent to clusters of ACR-12::GFP (Figure 2, A and B) (He et al. 2015; Howell et al. 2015). To ask if OIG-1 is also secreted when expressed from the native locus, we used CRISPR/Cas9 to engineer a single-copy knock-in of the TagRFP together with a 3XFLAG epitope tag (Figure 2B and Figure S2, A-C). We used immunoblotting to confirm expression of TagRFP::3XFLAG::OIG-1 (Figure 2C) but failed to detect TagRFP expression in vivo either by TagRFP fluorescence (Figure 2, D-G) or by immunostaining against the 3XFLAG epitope (data not shown). To produce a potentially brighter signal, we created a gfp $11_{x}:$ : oig-1 knock-in (Figure 2B) with a sgRNA that targeted the same $5^{\prime}-\mathrm{N}_{18}$ GGNGG site used for the TagRFP insert (Farboud and Meyer 2015). This strategy was designed to enhance a potential fluorescent signal by attaching seven copies of the GFP11 peptide to the OIG-1 N-terminus (Kamiyama et al. 2016). Successful knock-in of $g f p 11_{x 7}$ was confirmed by sequencing (data not shown). 
A
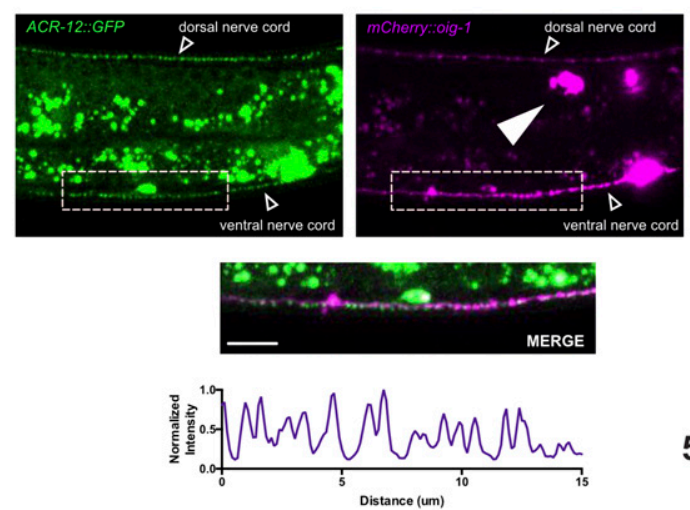
TagRFP

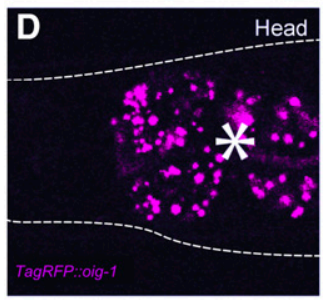

oig-1 native locus

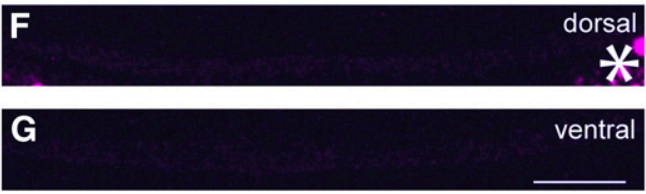

L
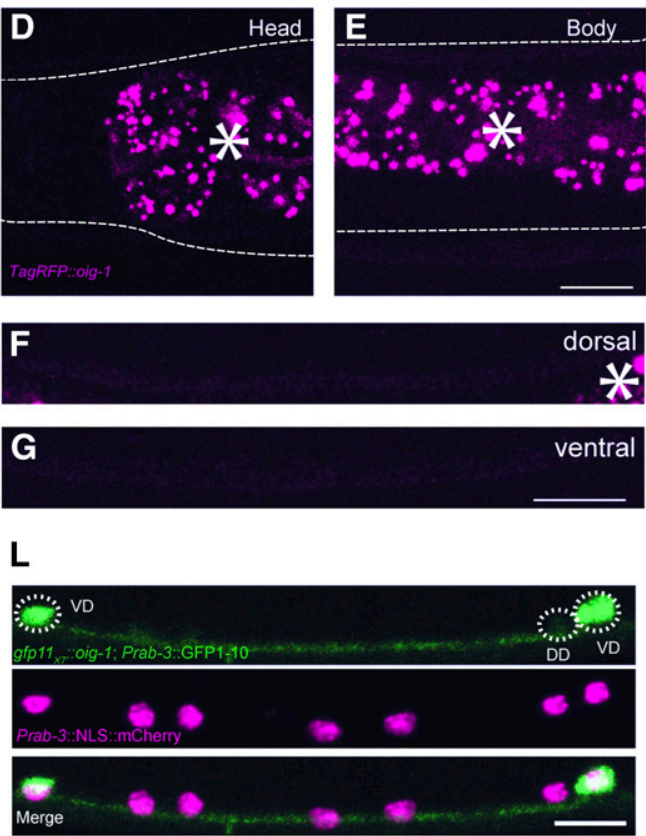

dorsal

entral
B

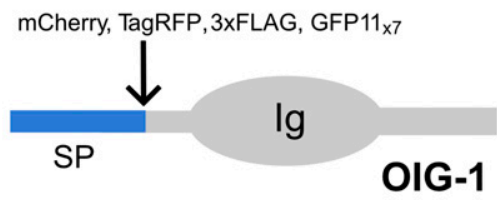

C

oig-1

oig-1 knock-in transgenic OE

$50 \mathrm{kDa}$

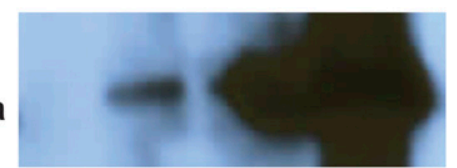

gfp 11 oig-1 native locus

\section{r}

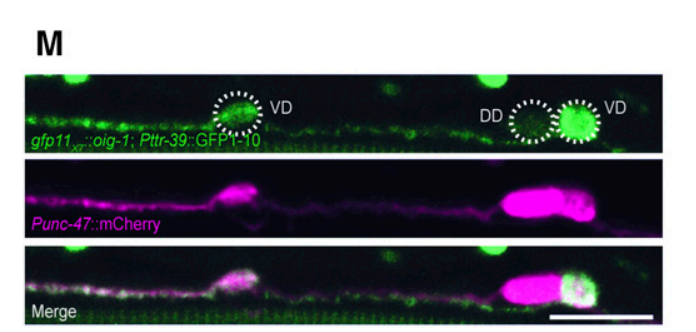

Figure 2 CRISPR knock-in of GFP1 $1_{x 7}$ in OIG-1 reveals its intracellular localization in GABAergic motor neurons. (A) Overexpression of mCherry::OIG-1 from a transgenic array (Punc-25::mCherry::oig-1) produces bright puncta (magenta) adjacent to ACR-12::GFP-labeled postsynaptic clusters (green) of AChRs in GABAergic motor neurons (Punc-47::acr-12::gfp). Merge shows ventral nerve cord. Bar, $10 \mu \mathrm{m}$. Line scan of mCherry:: OIG-1 in the ventral nerve cord (bottom) shows punctate signal. Arrowhead points to coelomocyte. (B) OIG-1 protein showing $\mathrm{N}$-terminal SP, C-terminal Ig (Immunoglobulin) domain, and insertion site for fluorescent and epitope tags (mCherry, TagRFP, 3xFLAG, and GFP11 7 ). (C) Immunoblot stained for the 3XFLAG tag detects expression of singlecopy TagRFP::OIG-1 knock-in and confirms over expression of mCherry::3XFLAG::OIG-1 from multicopy transgenic array. (D-G) TagRFP knock-in at the oig-1 locus (top) does not result in detectable TagRFP::OIG-1 fluorescence. Note absence of TagRFP::OIG-1 signal in head neurons (D), body $(\mathrm{E})$, and dorsal $(\mathrm{F})$ and ventral $(\mathrm{G})$ nerve cords. Asterisks mark autofluorescent granules. ( $\mathrm{H}-\mathrm{K}$ ) OIG-1 expression in the nervous system. The gfp $11_{x 7}$ :. oig-1 knock-in (top) was crossed with the panneural transgenic line expressing Prab-3::GFP1-10. A diffuse OIG-1 NATF GFP signal is detected in head neurons $(H)$ and in VD but not DD GABAergic motor neuron cell soma (dashed outlines) in the ventral cord (I). NATF GFP-labeled OIG-1 is detectable in both dorsal and ventral nerve cords (J and K). Asterisk marks autofluorescent granules. ( $\mathrm{L}$ and $\mathrm{M}$ ) OIG-1 expression in the nervous system and in GABAergic motor neurons. (L) The gfp1 $1_{x 7}: .$. oig-1 knock-in line was crossed with the pan-neural marker Prab-3::gfp1-10 and all neurons labeled with a nuclear-localized pan-neural label, Prab-3::NLS::mCherry. Note OIG-1 NATF GFP signal specifically in VD but not DD cell soma (dashed outlines), nor in additional ventral cord Prab-3::NLS::mCherry-labeled nuclei corresponding to cholinergic motor neurons. (M) The gfp11 $x$ :::oig-1 knock-in was crossed with the GABAergic motor neuron-specific marker Pttr-39::gfp1-10 and all GABA neurons were labeled with Punc-47::mCherry. Note the OIG-1 NATF GFP signal in VD but not DD motor neurons (dashed outlines) ${ }^{14}$. GFP1-10 is cytosolically expressed from the Prab-3::gfp 1-10 and Pttr-39::gfp1-10 transgenes, thus indicating that OIG-1 is intracellularly localized at its native expression level. All fluorescent images were obtained from L4-stage larvae. Bar, $10 \mu \mathrm{m}$. AChR, acetylcholine receptor; CRISPR, clustered regularly interspaced short palindromic repeats; DD, Dorsal D neuron; GABA, $\gamma$-aminobutyric acid; NATF, Native And Tissue-specific Fluorescence; SP, signal peptide; TagRFP, Tag red fluorescent protein; VD, Ventral D neuron.

We have previously shown that ACR-12::GFP in VD-class GABAergic motor neurons mislocalizes to the ventral side in oig-1 mutants, thereby resulting in an asymmetric ACR-12:: GFP signal that is brighter in the ventral $v s$. dorsal nerve cords (Figure S2) (He et al. 2015). In contrast, in the wildtype, ACR-12::GFP puncta are evenly distributed between dorsal and ventral nerve cords. This symmetry is maintained in the gfp $11_{X 7}:$ :oig-1 strain, thus arguing that the GFP $11_{x 7}$ adduct does not significantly disrupt OIG-1 function (Figure S2, C and D). We then crossed the $g f p 11_{x} ;:$ :oig-1 knock-in with a pan-neural
Prab-3::gfp1-10 transgenic line. Consistent with our previous findings, the OIG-1 NATF GFP signal can be detected in head neurons, and in both dorsal and ventral nerve cords (Figure 2, H-K) (He et al. 2015). Colocalization of OIG-1 NATF GFP with the nuclear-localized pan-neural marker Prab-3::NLS:: mCherry confirmed OIG-1 expression in neurons (Figure 2L). As an independent strategy to validate OIG-1 expression in GABA neurons, we crossed the $g f p 11_{x} ;:$ :oig-1 line with Pttr-39::gfp1-10, which is selectively expressed in DD- and VD-class GABAergic motor neurons (Cinar et al. 2005). In 
this case, the OIG-1 NATF GFP signal is limited to VD neurons with either weak or undetectable expression in DD neurons in L4 larvae (Figure 2M). This finding confirms previous results obtained with a Poig-1::gfp transcriptional reporter that was expressed in VD, but not DD, neurons after the L2 larval stage (He et al. 2015). Because the GFP1-10 peptide is expressed intracellularly in these strains, the NATF GFP signal likely derives from cytoplasmic OIG-1. Notably, the OIG-1 NATF GFP signal is visible throughout VD neuron soma and neurites (Figure 2, H-M), and does not show the distinctive highly punctate appearance of OIG-1 when overexpressed from a multicopy array (Figure 2A) (Figure S3) (He et al. 2015; Howell et al., 2015). To test for potential secretion of OIG-1 from the native locus, the $g f p 11_{x 7}$ ::oig- 1 knock-in was crossed with a transgenic line in which the GFP1-10 peptide is secreted from neurons (Prab-3::ss::gfp1-10). However, this experiment did not produce a detectable extracellular NATF signal nor GFP fluorescence in coelomocytes in the body cavity, which normally function as macrophage-like cells and thus can be used to detect secreted protein markers (Figure S4A) (Fares and Greenwald 2001). Notably, overexpression of mCherry:: OIG-1 from an extrachromosomal array does label coelomocytes (He et al. 2015; Howell et al. 2015). As a positive control, we showed that the secreted form of GFP1-10 in the Prab$3:: s s:: g f p 1-10$ strain is functional because it robustly labels a GFP11 peptide fused to the extracellular domain of the synaptic membrane protein NLG-1 (Feinberg et al. 2008) (Figure S4, B-D). As negative controls, we showed that neither GFP $11_{x 7}$ nor GFP1-10 by themselves produce visible GFP fluorescence (Figure S2, E and F). Although undetectably low levels of secreted OIG-1 could be produced by this experiment, our overall results are consistent with the hypothesis that OIG-1 is not secreted when expressed at the native level but localizes intracellularly (S. He, A. Cuentas Condori, D. Miller, unpublished data). For example, genetic disruption of the OIG-1 signal peptide blocks OIG-1 secretion but does not disrupt oig-1 function in vivo (He et al. 2015). Our finding that OIG is intracellularly localized depended on the use of the NATF strategy to reveal low levels of native OIG-1 expression and thereby circumvent artifactual extracellular localization due to OIG-1 overexpression from multicopy arrays (He et al. 2015).

\section{NATF GFP labeling reveals discrete locations for the transmembrane domain protein LEV-10 in different cell types}

Having shown that NATF could detect a soluble protein (OIG1), we next targeted LEV-10, a CUB domain transmembrane protein that clusters AChRs at postsynaptic sites in body muscles (Gally et al. 2004). First, we created a CRISPR/Cas9 knock-in line in which a single copy of GFP was fused to the intracellular C-terminus of LEV-10 (see Figure 4A). We detected LEV-10::GFP in both ventral and dorsal nerve cords, as predicted for a protein that localizes to body muscle synapses (Gally et al. 2004). LEV-10::GFP puncta were also detected in the head region where motor neurons synapse with body muscles on the inside surface of the nerve ring (White et al. 1986; Von Stetina et al. 2006) (Figure 3A). For NATF GFP labeling of body muscle synapses, we generated a lev$10:: g f p 11_{x 7}$ knock-in and crossed it with a muscle-specific line expressing GFP1-10 (Pmyo-3::gfp1-10) from an extrachromosomal array. The LEV-10 muscle-specific NATF GFP signal in the head region and axial nerve cords (Figure 3B) mimics that of the single-copy lev-10::gfp knock-in (Figure 3A), but is noticeably brighter. We quantified the GFP signal for each marker at the nerve ring muscle synapses to confirm that the LEV-10 NATF fluorescence is brighter (around three times) than the GFP signal from the lev-10::gfp single-copy insertion, as predicted from measurements of single-copy $v s$. multicopy split-GFP expressed in cultured cells (Figure 3C) (Kamiyama et al. 2016). In addition to determining that the lev-10::gfp $11_{x 7}$ array yields a stronger signal than that of the single-copy lev-10::GFP insert, we also showed that NATF GFP is substantially more resistant to photobleaching, as previously demonstrated for reconstituted split-GFP from measurements in vitro (Kamiyama et al. 2016) (Figure 3D).

In addition to expression in muscle, our independent studies have shown that LEV-10 is also expressed in ventral cord neurons where it colocalizes with AChRs at postsynaptic sites in GABAergic motor neurons (S. He, A. Cuentas Condori, D. Miller, unpulished data). In the motor neuron circuit, cholinergic motor neurons form dyadic synapses that innervate closely spaced postsynaptic domains in body muscle and GABA neurons (see Figure 4G) (White et al. 1986). Both of these postsynaptic regions in the ventral nerve cord region should be labeled in the lev-10::gfp knock-in and, thus, cannot be unambiguously identified (Figure 3A). To resolve this problem, we crossed the lev-10::gfp $11_{x 7}$ knock-in with transgenic lines that express GFP1-10 in either body muscles (Pmyo-3::gfp1-10), or in DD and VD GABAergic motor neurons (Pttr-39::gfp1-10). NATF GFP puncta can be readily detected in both cases (Figure 4, C and D), but are brighter in muscles than in GABAergic neurons (data not shown). Expression of a TagRFP-labeled AChR subunit UNC-29 (Gally et al. 2004) in muscle confirms colocalization of UNC-29::TagRFP with LEV-10 NATF GFP reconstituted in muscle. (Figure 4C). Expression of GFP1-10 in DD and VD neurons produces LEV-10 NATF GFP puncta that overlap with a cytoplasmic GABA neuron mCherry marker (Punc$47::$ mCherry), as predicted for the LEV-10 protein that localizes to GABA neuron synapses (Figure 4D). To confirm the postsynaptic location of LEV-10 in GABA neurons, we used a DD-specific construct (Pflp-13::gfp1-10) to generate a LEV-10 NATF GFP signal. In this case, superresolution imaging resolves distinct LEV-10 NATF GFP puncta at the tips of postsynaptic spine-like projections that have been recently described in the ventral processes of mature DD neurons (Figure 4E) (Philbrook et al. 2018). Notably, we have also observed that the AChR marker, ACR-12::GFP, is positioned in the same distal location in DD dendritic spines and that these spines are aligned with presynaptic cholinergic vesicles (Cuentas-Condori et al. 2019) (Philbrook et al. 2018). In addition to resolving 
A

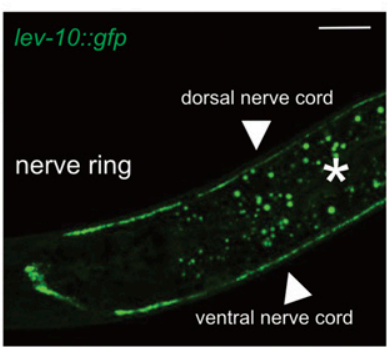

B
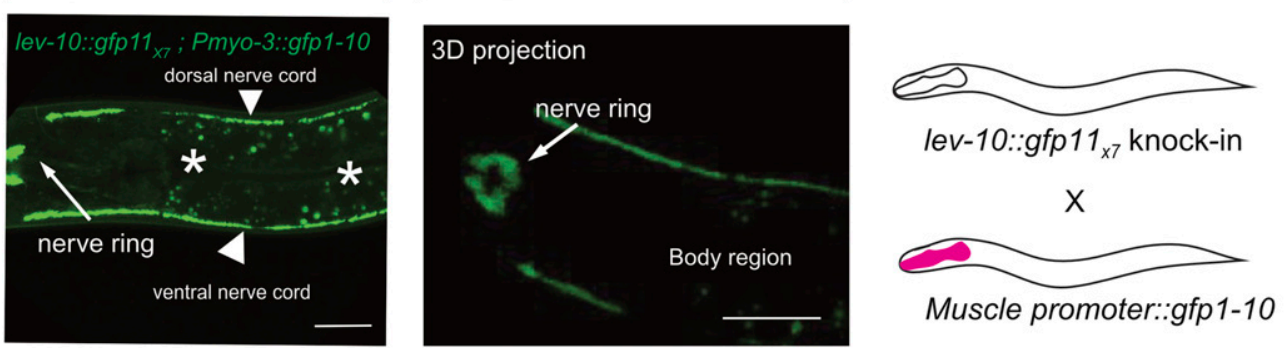

C

Brightness

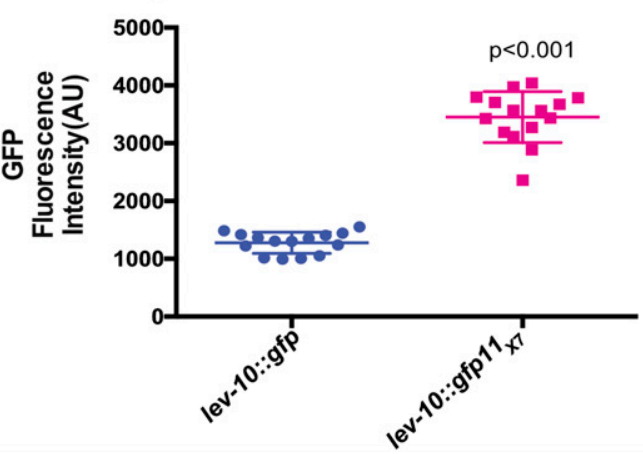

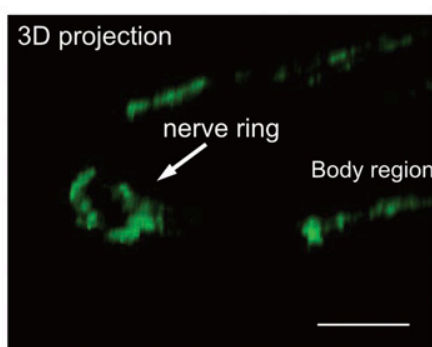

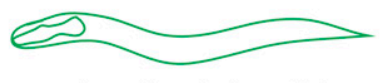

lev-10::gfp knock-in

the nerve ring $(0.45 \pm 0.12), N=10, P<0.001$, Student's $t$-test. See Materials and Methods. Error bars are SD. AU, arbitrary units; NATF, Native And Tissue-specific Fluorescence.

LEV-10 localization at distinct postsynaptic locations in muscle vs. GABA neurons, we also used a cholinergic motor neuron driver (Pacr-2::gfp1-10) to detect a separate LEV-10 NATF signal in ventral cord cholinergic neurons. In this case, LEV-10 NATF GFP is diffuse (Figure 4F) and asymmetrically localized to the ventral, but not dorsal, nerve cord (data not shown), a labeling pattern that closely resembles the perisynaptic position of the AChR subunit ACR-12::GFP in cholinergic motor neurons (Petrash et al. 2013). Because LEV-10 is expressed at its native level and retains its AChR clustering function (data not shown) when fused to the GFP $11_{\mathrm{X} 7}$ adduct, it seems likely that each of the three distinct, cell-specific LEV-10 NATF signals (i.e., muscle, GABA neurons, and cholinergic neurons) marks authentic subcellular locations for the endogenously expressed LEV-10 protein.

\section{Discussion}

We have shown that NATF offers a robust strategy for producing bright, cell-specific signals for the C. elegans proteins OIG-1 and LEV-10 expressed from their native genomic loci. These results suggest that NATF should be especially useful
Figure 3 lev-10::gfp $11_{x 7}$ yields a stronger NATF GFP signal than the single-copy lev-10::gfp knock-in at synapses in neurons and muscle cells. (A) Confocal image showing localization of LEV-10::GFP in a single-copy GFP knock-in at the native lev-10 gene (lev-10::gfp). LEV-10::GFP puncta are visible at the nerve ring (arrow), and in ventral and dorsal nerve cords (arrowheads). (B) Confocal image of the LEV-10 NATF GFP signal at body muscle synapses arising from the combination of the lev10::gfp $11_{x}$ knock-in with Pmyo$3::$ gfp 1-10. NATF GFP (arrow) is detected at neuromuscular synapses near the nerve ring. Bar, 20 $\mu \mathrm{m}$. Insets (right) shows rotated views of anterior regions of images on left to depict nerve ring labeling. Asterisks mark gut autofluorescence. (C) LEV-10 NATF GFP at body muscle synapses in the nerve ring labeled with lev-10::gfp $11_{x>}$ is significantly brighter (around three times) $(3450 \pm 441)$ than the single-copy lev-10::gfp knock-in $(1280 \pm 184) . P<0.001$, $N=15$, Student's $t$-test. Error bars are SD. (D) The LEV-10 NATF GFP signal at body muscle synapses in the nerve ring labeled with lev-10::gfp $11_{X 7}$ is significantly more stable (0.75 \pm $0.15)$ to photobleaching than the single-copy lev-10::gfp knock-in in

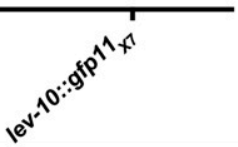

for marking connections in the compact $C$. elegans nervous system, where most synapses are located in the densely packed nerve ring and axial nerve cords (White et al. 1986). For example, GFP-tagging of a core presynaptic protein (e.g., RAB-3) by conventional CRISPR/Cas9 editing should mark synapses throughout the nervous system. In contrast, labeling with the NATF strategy should result in a bright, photostable GFP signal that is limited to the presynaptic domains of specific neurons. Although our results have determined that fusion with the GFP11 1 x peptide does not result in detectable disruption of the in vivo function of either OIG-1 (Figure S2, B-D) or LEV-10 (Figure 4C and S. (S. He, A. Cuentas Condori, D. Miller, unpublished data), other proteins may be less tolerant. In that event, smaller adducts with fewer copies of GFP11 could be attempted. In that case, GFP signal augmentation will be diminished but tissue-specific labeling is still possible (Noma et al. 2017). Because the $g f p 11_{X 7}$ insert is stably integrated at the native locus and is thus limiting, the complementing GFP1-10 peptide can be provided from multicopy transgenic arrays without risk of inducing overexpression artifacts. Thus, a given GFP $11_{X 7}$ split GFP insert can be rapidly tested with multiple tissue-specific GFP1-10 transgenic lines, 


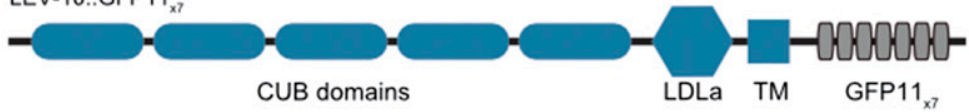

B

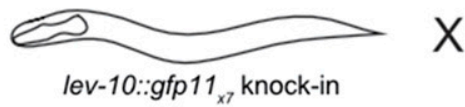

C

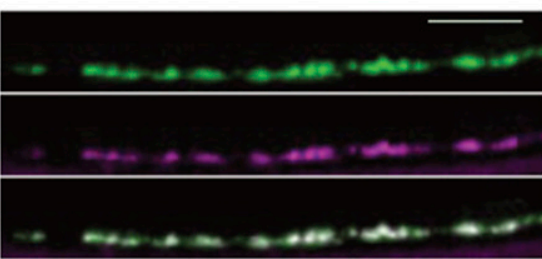

D

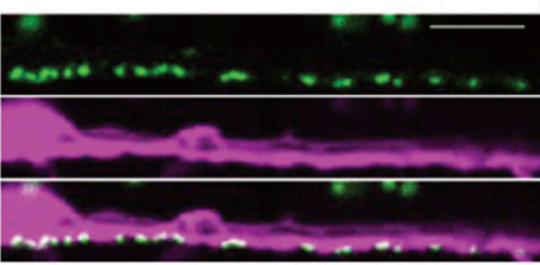

E

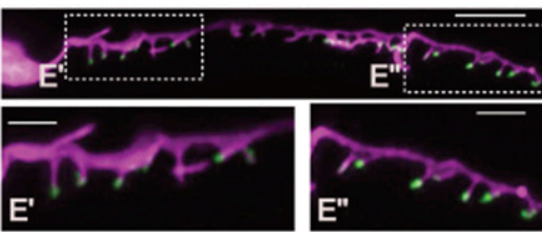

$\mathbf{F}$
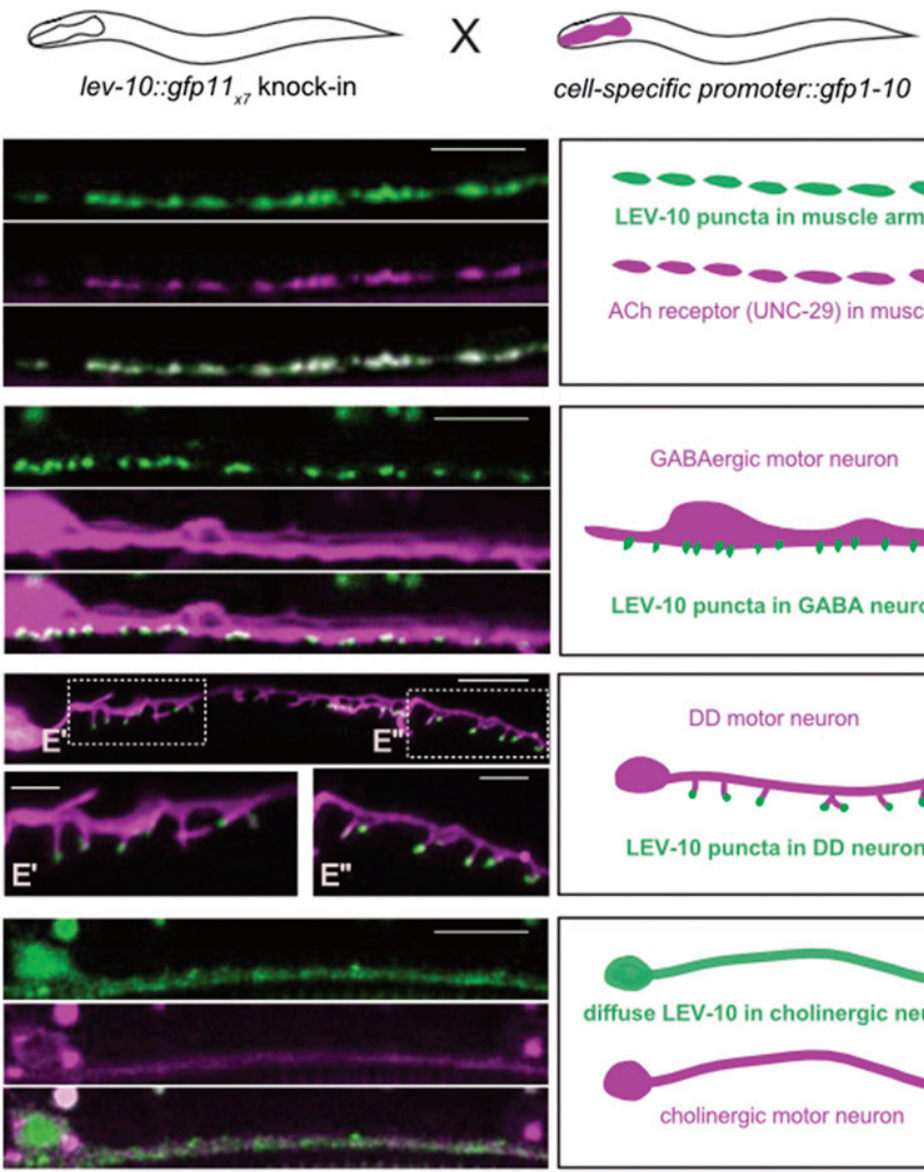

cell-specific promoter::gfp1-10
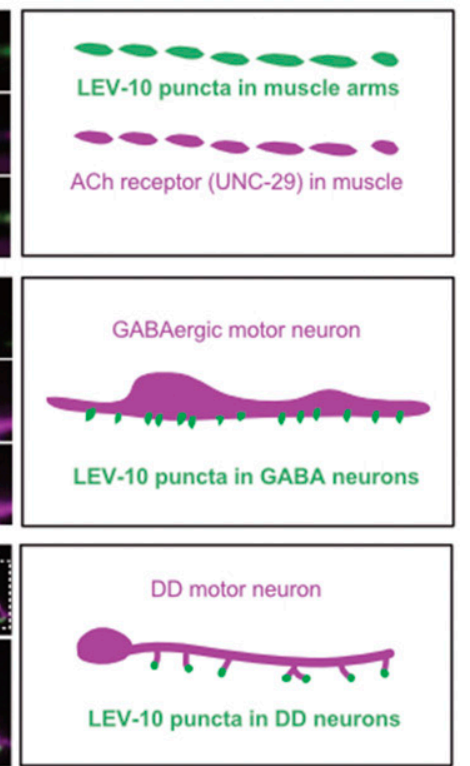

G

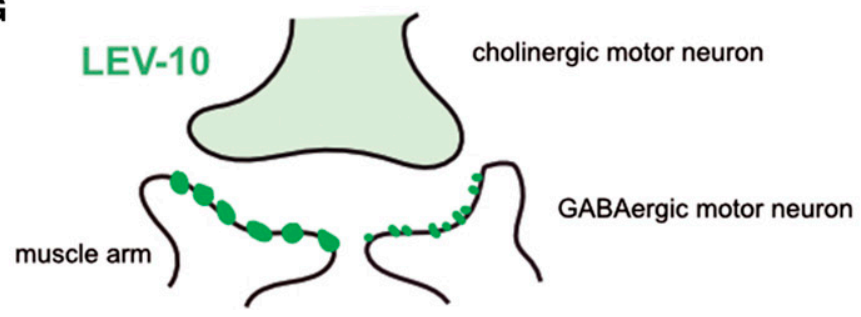

Figure 4 Visualization of LEV-10 NATF GFP signal at cellspecific synapses. (A) Schematic of LEV-10::GFP11 $x 7$ showing extracellular complement $\mathrm{C} 1 \mathrm{r} / \mathrm{C} 1 \mathrm{~s}$, Uegf, Bmp1 (CUB) CUB and low-density lipoprotein receptor domain class $A$ (LDLa) LDLa domains, with TM region and cytoplasmic tail with GFP1 $1_{x 7}$ insert. (B) Cell-specific labeling strategy. The lev-10::gfp $11_{x 7}$ knock-in strain is crossed with separate transgenic lines expressing GFP1-10 in specific cell types. (C-F) Representative images (left) and schematics (right) of ventral nerve cord region of L4 larvae showing LEV-10 NATF GFP arising from complementation of the lev-10:: gfp $11_{x 7}$ knock-in with cell-specific expression of GFP1-10: (C) Pmyo-3::gfp 1-10 (body muscle), (D) Pttr-39::gfp 1-10 (DD and VD GABAergic motor neurons), (E) Pflp-13::gfp1-10 (DD GABAergic motor neurons) with superresolution images with insets $\left(E^{\prime}\right.$ and $\left.E^{\prime \prime}\right)$ showing localization of LEV-10 NATF GFP to tips of postsynaptic DD dendritic spines, and (F) Pacr-2::gfp 1-10 (cholinergic motor neurons). Pmyo-3::unc-29:TagRFP marks ACh receptors in body muscle in (C), Punc-47::mCherry labels GABA neurons in (D), Pflp-13::LifeAct::mCherry marks DD neurons in (E), and Punc-4::mCherry labels cholinergic motor neurons in (F). Bars, $5(\mathrm{C}-\mathrm{F})$ and $2 \mu \mathrm{m}$ ( $\mathrm{E}^{\prime}$ and $\left.\mathrm{E}^{\prime \prime}\right)$. (G) Schematic showing distribution of LEV-10 NATF GFP at a dyadic synapse of a presynaptic cholinergic motor neuron with postsynaptic body muscle and GABAergic motor neuron in the ventral nerve cord. ACh, acetylcholine; DD, Dorsal D neuron; GABA, $\gamma$-aminobutyric acid; NATF, Native And Tissuespecific Fluorescence; TagRFP, Tag red fluorescent protein; $T M$, transmembrane; VD, Ventral $D$ neuron. which can be readily generated using conventional methods. A similar combinatorial approach should also be useful for tissue-specific protein labeling in other model organisms (Kelliher et al. 2018). We note that NATF can be modified to reduce weak background fluorescence from the GFP1-10 fragment (Feng et al. 2017), and for multicolor split-GFP imaging with cyan (CFP) and yellow (YFP) GFP variants, or with the sfmCherry marker (Kamiyama et al. 2016; Feng et al. 2017).

\section{Acknowledgments}

We thank members of the Miller laboratory for critical reading of the manuscript, Sierra Palumbos and Alice Siqi Chen for plasmid construction, Lakshmi Sundararajan for help with confocal imaging, and Oliver Hobert for sharing strains. Some nematode strains used in this work were provided by the Caenorhabditis Genetics Center, which is funded by the National Institutes of Health (NIH) National Center for Research Resources. Superresolution images were acquired in the Vanderbilt Cell Imaging Shared Resource (1S10 OD-201630-01). This work was supported by NIH grants to D.M.M. (R01 NS-081259 and R01 NS-106951). A.C.-C. is supported by an American Heart Association predoctoral fellowship (18PRE33960581).

Author contributions: S.H., A.C.-C., and D.M.M. conceived the project. S.H. and A.C.-C. performed the experiments and analyzed the data. S.H., A.C.-C., and D.M.M. wrote the manuscript. The authors declare no competing interests. 


\section{Literature Cited}

Alegria-Schaffer, A., A. Lodge, and K. Vattem, 2009 Performing and optimizing Western blots with an emphasis on chemiluminescent detection. Methods Enzymol. 463: 573-599. https:// doi.org/10.1016/S0076-6879(09)63033-0

Brenner, S., 1974 The genetics of Caenorhabditis elegans. Genetics 77: 71-94.

Cabantous, S., T. C. Terwilliger, and G. S. Waldo, 2005 Protein tagging and detection with engineered self-assembling fragments of green fluorescent protein. Nat. Biotechnol. 23: 102107. https://doi.org/10.1038/nbt1044

Chalfie, M., 2009 GFP: lighting up life. Proc. Natl. Acad. Sci. USA 106: 10073-10080. https://doi.org/10.1073/pnas.0904061106

Cinar, H., S. Keles, and Y. Jin, 2005 Expression profiling of GABAergic motor neurons in Caenorhabditis elegans. Curr. Biol. 15: 340-346. https://doi.org/10.1016/j.cub.2005.02.025

Cuentas-Condori, A., B. Mulcahy, S. He, S. Palumbos, M. Zhen et al., 2019 Dendritic spines on GABAergic neurons respond to cholinergic signaling in the Caenorhabditis elegans motor circuit. bioRxiv. Available at: https://www.biorxiv.org/content/ $10.1101 / 598714 \mathrm{v} 2$.

Dickinson, D. J., A. M. Pani, J. K. Heppert, C. D. Higgins, and B. Goldstein, 2015 Streamlined genome engineering with a selfexcising drug selection cassette. Genetics 200: 1035-1049. https://doi.org/10.1534/genetics.115.178335

Duerr, J. S., 2006 Immunohistochemistry (June 19, 2006), WormBook ed. The C. elegans Research Community, WormBook, doi/10.1895/wormbook.1.105.1, http://www.wormbook.org.

El Mouridi, S., C. Lecroisey, P. Tardy, M. Mercier, A. Leclercq-Blondel et al., 2017 Reliable CRISPR/Cas9 genome engineering in Caenorhabditis elegans using a single efficient sgRNA and an easily recognizable phenotype. G3 (Bethesda) 7: 1429-1437. https:// doi.org/10.1534/g3.117.040824

Evans, T. C., 2006 Transformation and microinjection (April 6, 2006), WormBook, ed. The C. elegans Research Community, WormBook, doi/10.1895/wormbook.1.108.1, http://www.wormbook.org.

Farboud, B., and B. J. Meyer, 2015 Dramatic enhancement of genome editing by CRISPR/Cas9 through improved guide RNA design. Genetics 199: 959-971. https://doi.org/10.1534/ genetics.115.175166

Fares, H., and I. Greenwald, 2001 Genetic analysis of endocytosis in Caenorhabditis elegans: coelomocyte uptake defective mutants. Genetics 159: 133-145.

Feinberg, E. H., M. K. Vanhoven, A. Bendesky, G. Wang, R. D. Fetter et al., 2008 GFP reconstitution across synaptic partners (GRASP) defines cell contacts and synapses in living nervous systems. Neuron 57: 353-363. https://doi.org/10.1016/j.neuron.2007.11.030

Feng, S., S. Sekine, V. Pessino, H. Li, M. D. Leonetti et al., 2017 Improved split fluorescent proteins for endogenous protein labeling. Nat. Commun. 8: 370. https://doi.org/10.1038/ s41467-017-00494-8

Gally, C., S. Eimer, J. E. Richmond, and J.-L. Bessereau, 2004 A transmembrane protein required for acetylcholine receptor clustering in Caenorhabditis elegans. Nature 431: 578-582. https:// doi.org/10.1038/nature02893

He, S., A. Philbrook, R. McWhirter, C. V. Gabel, D. G. Taub et al., 2015 Transcriptional control of synaptic remodeling through regulated expression of an immunoglobulin superfamily protein. Curr. Biol. 25: 2541-2548. https://doi.org/10.1016/ j.cub.2015.08.022
Hostettler, L., L. Grundy, S. Käser-Pébernard, C. Wicky, W. R. Schafer et al., 2017 The bright fluorescent protein $\mathrm{mNeonGreen} \mathrm{facilitates}$ protein expression analysis in vivo. G3 (Bethesda) 7: 607-615. https://doi.org/10.1534/g3.116.038133

Howell, K., J. G. White, and O. Hobert, 2015 Spatiotemporal control of a novel synaptic organizer molecule. Nature 523: 83-87. https://doi.org/10.1038/nature14545

Hsu, P. D., E. S. Lander, and F. Zhang, 2014 Development and applications of CRISPR-Cas9 for genome engineering. Cell 157: 1262-1278. https://doi.org/10.1016/j.cell.2014.05.010

Kamiyama, D., S. Sekine, B. Barsi-Rhyne, J. Hu, B. Chen et al., 2016 Versatile protein tagging in cells with split fluorescent protein. Nat. Commun. 7: 11046. https://doi.org/10.1038/ ncomms11046

Kelliher, M. T., Y. Yue, A. Ng, D. Kamiyama, B. Huang et al., 2018 Autoinhibition of kinesin-1 is essential to the dendritespecific localization of Golgi outposts. J. Cell Biol. 217: 25312547. https://doi.org/10.1083/jcb.201708096

Miller, D. M., I. Ortiz, G. C. Berliner, and H. F. Epstein, 1983 Differential localization of two myosins within nematode thick filaments. Cell 34: 477-490. https://doi.org/10.1016/ 0092-8674(83)90381-1

Noma, K., A. Goncharov, M. H. Ellisman, and Y. Jin, 2017 Microtubule-dependent ribosome localization in C. elegans neurons. Elife 6: e26376. https://doi.org/10.7554/ eLife.26376

Petrash, H. A., A. Philbrook, M. Haburcak, B. Barbagallo, and M. M. Francis, 2013 ACR-12 ionotropic acetylcholine receptor complexes regulate inhibitory motor neuron activity in Caenorhabditis elegans. J. Neurosci. 33: 5524-5532. https://doi.org/ 10.1523/JNEUROSCI.4384-12.2013

Philbrook, A., S. Ramachandran, C. M. Lambert, D. Oliver, J. Florman et al., 2018 Neurexin directs partner-specific synaptic connectivity in C. elegans. Elife 7: e35692. https://doi.org/10.7554/ eLife.35692

Praitis, V., E. Casey, D. Collar, and J. Austin, 2001 Creation of lowcopy integrated transgenic lines in Caenorhabditis elegans. Genetics 157: 1217-1226.

Remington, S. J., 2011 Green fluorescent protein: a perspective. Protein Sci. 20: 1509-1519. https://doi.org/10.1002/pro.684

Schwartz, M. L., and E. M. Jorgensen, 2016 SapTrap, a toolkit for high-throughput CRISPR/Cas9 gene modification in Caenorhabditis elegans. Genetics 202: 1277-1288. https://doi.org/ 10.1534/genetics.115.184275

Tanenbaum, M. E., L. A. Gilbert, L. S. Qi, J. S. Weissman, and R. D. Vale, 2014 A protein-tagging system for signal amplification in gene expression and fluorescence imaging. Cell 159: 635-646. https://doi.org/10.1016/j.cell.2014.09.039

Terpe, K., 2003 Overview of tag protein fusions: from molecular and biochemical fundamentals to commercial systems. Appl. Microbiol. Biotechnol. 60: 523-533. https://doi.org/10.1007/ s00253-002-1158-6

Von Stetina, S. E., M. Treinin, and D. M. Miller, III, 2006 The motor circuit. Int. Rev. Neurobiol. 69: 125-167. https:// doi.org/10.1016/S0074-7742(05)69005-8

White, J. G., E. Southgate, J. N. Thomson, and S. Brenner, 1986 The structure of the nervous system of the nematode Caenorhabditis elegans. Philos. Trans. R. Soc. Lond. B Biol. Sci. 314: 1-340.

Communicating editor: O. Hobert 